\title{
Melanoma maligno conjuntival
}

\section{Malignant melanoma of the conjunctiva}

\author{
Gustavo Amorim Novals ${ }^{1}$, Carol Lynn Karp ${ }^{2}$
}

\section{RESUMO}

Introdução: Os tumores melanocíticos conjuntivais compreendem lesões que podem variar desde lesões benignas como os nevos conjuntivais, lesões pré-cancerosas como melanose adquirida primária com atipia até o melanoma maligno conjuntival. O reconhecimento das características clinicas destas lesões e seu diagnóstico preciso permitem o tratamento adequado, contribuindo para a redução da morbidade e mortalidade associados ao melanoma de conjuntiva. Objetivo: Revisão das características clinicas, diagnóstico e modalidades de tratamento clínico e cirúrgico das lesões precursoras (Nevos e melanose adquirida primária) e do maligno conjuntival.

Métodos: Revisão de literatura através de pesquisa no banco de dados MEDLINE PUBMED, LILACS e SciELO no período de 1980 a 2011. As palavras-chave utilizadas, individualmente ou em conjunto, foram:"conjunctival melanoma","primary acquired melanosis","nevi","treatment","chemotherapy","recurrence","metastasis" "emortality".

Resultado: Características clínicas que permitem o diagnóstico do melanoma conjuntival e sua diferenciação de outras lesões pigmentadas conjuntivais foram consideradas. O estadiamento clínico e patológico, assim como modalidades de tratamento para o melanoma maligno foram revisadas.

Conclusões: Pacientes portadores de lesões pigmentadas conjuntivais devem ser avaliados por um oftalmologista especialista. A história oftalmológica, a história familiar de melanoma, e características clínicas da lesão necessitam de cuidadosa avaliação, incluindo-se a determinação do risco de malignidade. A documentação fotográfica deve ser realizada. O tratamento clínico e planejamento cirúrgico devem ser baseados na suspeita clinica. A análise histopatológica por patologista experiente é fundamental para orientação do tratamento e para identificação de fatores prognósticos, principalmente em casos de doença maligna.

Descritores: Neoplasias da túnica conjuntiva/cirurgia; Neoplasias da túnica conjuntiva/mortalidade; Neoplasias da túnica conjuntiva/secundária; Melanoma/patologia; Melanose/patologia; Pigmentação/fisiopatologia; Diagnóstico diferencial; Nevos e melanoma; Estadiamento de neoplasias

\section{ABSTRACT}

Introduction: Melanocytic tumors comprise a wide range of lesions. These lesions can be nevi, primary acquired melanosis with or without atypia or malignant as conjunctival melanoma. Early recognition of the clinical features allows precise diagnosis and adequate intervention, reducing the rates of metastatic disease and mortality associated with conjunctival melanoma.

Purpose: To review clinicalfeatures, diagnosis, clinical and surgical treatment modalities of the different precursor pigmented lesions (Nevi and primary acquired melanosis) and malignant melanoma.

Methods: Literature review was performed accessing the database of MEDLINE, PUBMED, LILACS S SCIELO between 1980 and 2011. The keywords used, individually or combined, were: conjunctival melanoma, primary acquired melanosis, nevi, treatment, chemotherapy, recurrence, metastasis e mortality.

Results: Relevant clinical information for the diagnosis of malignant melanoma and differentiation from other pigmented conjunctival lesions were accessed. Clinical and pathological features and different modalities of treatment for malignant melanoma have been reviewed.

Conclusions: Patients presenting with a pigmented conjunctival lesion should be evaluated by an experienced ophthalmologist. Ocular history, family history of melanoma, and clinical features of the lesion need careful assessment and malignancy risk evaluation. Digital photo documentation should be performed. Clinical or surgical planning should be done based on the clinical suspicion. Careful histopathology analysis is required for treatment orientation and prognostic factors identification, mainly in cases of malignancy.

Keyword: Conjunctival neoplasms/surgery; Conjunctival neoplasms/mortality; Conjunctival neoplasms/secondary; Melanoma/pathology; Melanosis/pathology; Pigmentation/physiology; Nevi and melanomas; Diagnosis, differential; Neoplasm staging

\section{INTRODUÇÃO}

O melanoma maligno conjuntival (MC), apesar de raro, representa a segunda lesão maligna conjuntival mais frequente depois do carcinoma de células escamosas ${ }^{(1)}$. No passado, sua evolução resultava quase invariavelmente em prognóstico desfavorável, resultando em exenteração orbitária na tentativa de erradicação da doença altamente invasiva. Devido a sua baixa incidência, oftalmologistas geralmente não estão familiarizados e preparados para identificar e conduzir os casos suspeitos. O MC representa um desafio para o clínico e para o patologista, pois pode apresentar diversas facetas e originar-se de lesões aparentemente benignas como os nevos conjuntivais, ou até mesmo de lesões não pigmentadas como em alguns casos de melanose adquirida primária (MAP) sine pigmento ${ }^{(2)}$ O conhecimento das características do melanoma de conjuntiva, de suas lesões precursoras e dos sinais de transformação maligna são determinantes na identificação e na intervenção cirúrgica precoce, interferindo na história natural da doença e possibilitando a redução das taxas de recorrência, metástases e mortalidade.

\section{LESÕES PRECURSORAS}

\section{NeVo CONJUNTIVAL}

Os nevos conjuntivais são os tumores benignos mais frequentes da conjuntiva ${ }^{(3)}$. Os nevos podem ser congênitos ou adquiridos ${ }^{(4)}$.

Os nevos conjuntivais adquiridos costumam aparecer na primeira década de vida e são classificados de acordo com sua localização histológica em: juncional, composto e subepitelial. Acredita-se que esta classificação represente o espectro evolutivo dos nevos em seus
Submetido para publicação: 18 de maio de 2012

Aceito para publicação: 14 de julho de 2012

Trabalho realizado em Miami, no Bascom Palmer Eye Institute.

Médico, Universidade Federal do Estado do Rio de Janeiro - UNI-RIO - Rio de Janeiro (RJ), Brasil.

${ }^{2}$ Médica, Bascom Palmer Eye Institute, University of Miami, Miami, Florida (EUA).
Financiamento: Não houve financiamento para este trabalho.

Divulgação de potenciais conflitos de interesse: G.A.Novais, Nenhum; C.L.Karp, Nenhum.

Endereço para correspondência: Gustavo Novais, Rua Jeronyma Mesquita, 154 - Rio de Janeiro (RJ) - 22640-110 - Brasil - E-mail: gustavonovais@hotmail.com 
diferentes estágios na maturação e proliferação dos melanócitos, com atividade juncional no início e posterior migração das células névicas para a substância própria da conjuntiva ${ }^{(4)}$.

A região justalimbar da conjuntiva bulbar é o local mais comumente acometido, seguido pela região caruncular ${ }^{(5,6)}$. O acometimento da conjuntiva forniceal e tarsal é raro e seu envolvimento pode indicar maior risco de malignidade. A córnea não costuma ser acometida ${ }^{(5)}$.

Os nevos podem ser focais ou difusos, mas não multifocais. Por serem superficiais podem ser mobilizados com a conjuntiva sobre a esclera, a não ser quando situados sobre o limbo ou a conjuntiva palpebral(4). A grande maioria dos nevos conjuntivais é pigmentada e esta pigmentação pode variar aumentando sua intensidade na puberdade ${ }^{(7)}$ e na gestação(8). Ausência de pigmentação (nevos amelanóticos) é observada em 16 a 29\% dos $\operatorname{casos}^{(3,5)}$

Os nevos conjuntivais possuem margens bem delimitadas e vasos nutridores, embora menos frequentes que no MC, podem ser encontrados. A presença de cistos sugere benignidade e pode ser vista em até $65 \%$ dos nevos ${ }^{(5)}$. Na presença de pigmentação plana adjacente deve-se considerar a coexistência de melanose primária adquirida ${ }^{(4)}$.

Os nevos conjuntivais localizados na conjuntiva forniceal e tarsal devem ser excisados pelo maior risco de transformação maligna. Nevos localizados na conjuntiva bulbar (incluindo os situados no limbo e carúncula) com crescimento documentado, neovascularização ou vasos nutridores, presença de inflamação e aumento ou mudança no padrão de pigmentação devem ser excisados. Nevos recidivantes também devem ser excisados. Todo tecido deve ser encaminhado para avaliação histopatológica ${ }^{(9)}$. Também são causas de exérese a preocupação do paciente com melanoma conjuntival e com a aparência cosmética ${ }^{(5)}$.

\section{Melanose AdQUiRIDA PRIMÁRIA}

A melanose adquirida primária (MAP) da conjuntiva é uma lesão pigmentada plana, de aspecto amarronzado, variando sua tonalida- de de marrom-ouro a marrom-chocolate. É quase sempre unilateral ocorrendo em indivíduos da raça branca ${ }^{(10)}$. Tipicamente acomete pacientes de meia idade com predileção pelo sexo feminino ${ }^{(6.11)}$.

O termo "melanose" indica que o pigmento encontrado é derivado especificamente da produção de melanina, enquanto o termo "adquirida" distingue estas lesões das congênitas. O termo "primária" caracteriza que as lesões em questão não são decorrentes de processos generalizados de pigmentação, como em doenças sistêmicas (por exemplo, doença de Addison), ou secundário a fatores locais tais como a presença de corpo estranho, trauma, inflamação, ou uso de medicação tópica como epinefrina ${ }^{(12)}$.

Apesar de a conjuntiva bulbar ser o local mais frequentemente acometido pela MAP, todas as porções da conjuntiva podem ser afetadas. Quando há envolvimento da conjuntiva palpebral, a pigmentação pode estender-se sobre a margem palpebral até a epiderme adjacente. O envolvimento do epitélio corneano periférico também pode ser observado(10)

O aspecto clínico da MAP pode variar. Classicamente a área de pigmentação é difusa, pobremente circunscrita, irregular e localizada próxima ao limbo. As áreas de pigmentação podem diminuir de tamanho, permanecer estáveis ou exibir crescimento radial, intensificando ou diminuindo sua coloração. Pacientes sendo acompanhados por, ou com diagnóstico recente de MAP, e que apresentem alteração do padrão plano da lesão com o surgimento de um ou mais nódulos, devem ser considerados em risco de progressão para $\mathrm{MC} C^{(10)}$.

A MAP deve ser diferenciada de outras lesões pigmentadas, como os nevos juncionais, melanose racial benigna, melanose ocular ou oculodermal congênita, causas de pigmentação sistêmica (como doença de Addison), depósitos de maquiagem e de drogas tópicas (como depósitos de adenocromo da epinefrina) ${ }^{(13)}$.

A MAP pode ser classificada histologicamente em MAP com ou sem atipia. Esta diferenciação é fundamental, já que os pacientes com atipia apresentam risco de até $46,4 \%$ de progressão para melanoma
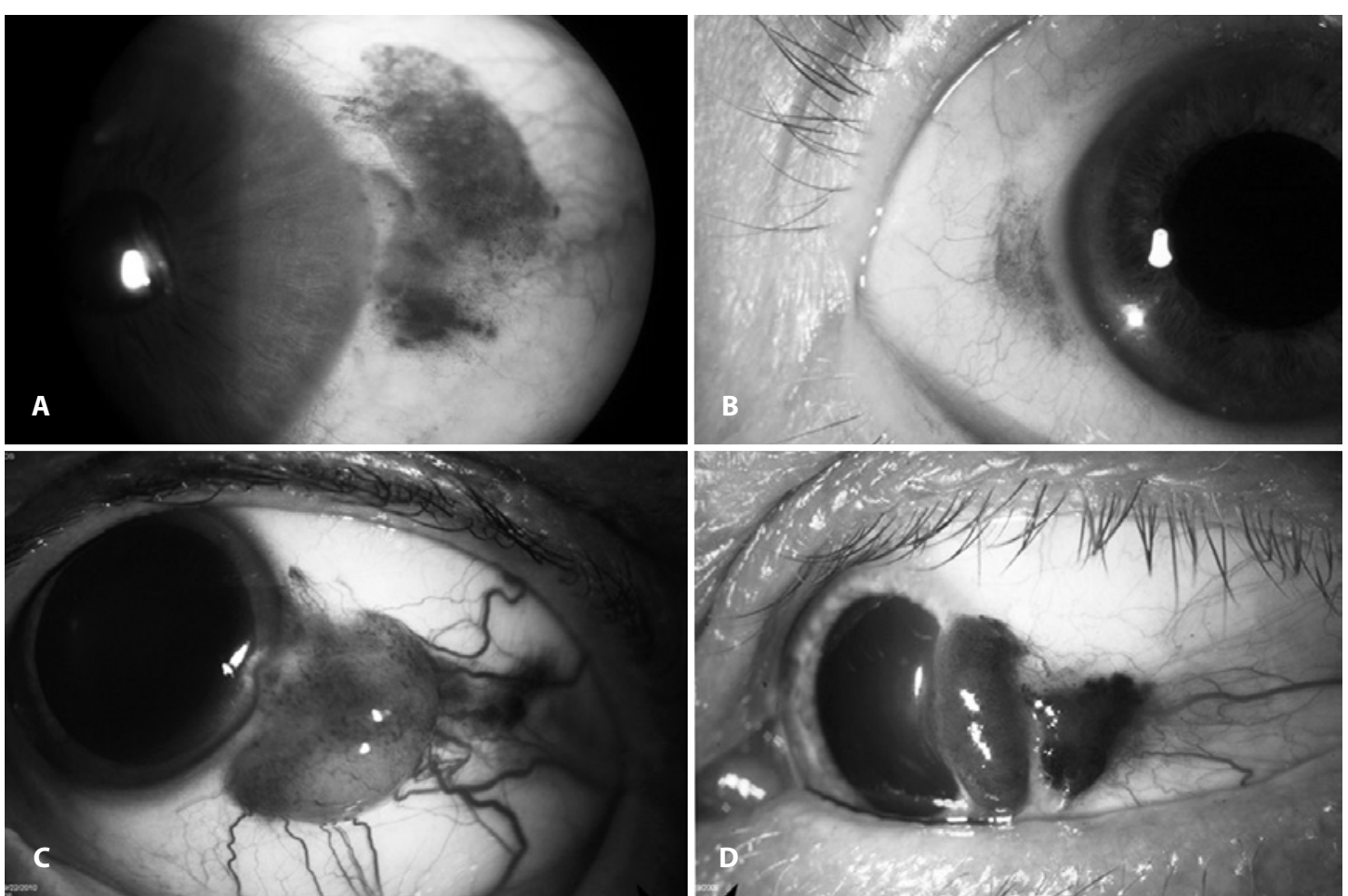

Figura 1. Lesões melanocíticas conjuntivais. A) Nevo conjuntival na região limbar com presença característica de cistos. B) Melanose adquirida primária na região limbar com pigmentação plana e bordas mal definidas. C e D) Melanoma conjuntival na região limbar. Observe a presença de vasos nutridores proeminentes e o aspecto nodular da lesão. O acometimento corneano pode ser visto (D). 
de conjuntiva (MC) em cerca de 2,5 anos ${ }^{(14)}$. Shields et al., observaram que de 311 pacientes com MAP, nenhum (0\%) dos que apresentavam lesões com atipia ausente ou leve evoluíram para MC, enquanto 13\% dos pacientes com atipia severa desenvolveram MC em pelo menos três anos de acompanhamento. Quanto maior a extensão em "horas de relógio" maior a chance de recorrência e progressão para $M C^{(11)}$. Pacientes portadores de MAP com atipia e aumento da densidade linfática tumoral apresentam maiores chances de recorrência, aumentando o risco de progressão para $M C^{(15)}$.

\section{Melanoma}

Apesar de raro, o melanoma conjuntival representa a segunda lesão maligna mais comum da conjuntiva ${ }^{(1)}$. O MC corresponde a apenas $1,6 \%$ de todos os melanomas não cutâneos ${ }^{(16)}$ e a $2 \%$ de todas as lesões malignas oculares ${ }^{(17)}$.

\section{INCIDÊNCIA}

O MC acomete com maior frequência indivíduos da raça branca com incidência variando de 0,24 a 0,80 por milhão de habitantes ${ }^{(18,19)}$. Sua incidência vem aumentando nos Estados Unidos da América entre a população masculina ${ }^{(20,21)}$ e em pacientes acima de 60 anos $^{(21)}$

MC é mais frequente em indivíduos idosos com idade média variando de 55 a 70 anos(6,18,19,22-25). Embora raro em jovens, existem relatos de casos de $\mathrm{MC}$ em pacientes com menos de 20 anos de idade ${ }^{(26,27)}$. Não existe diferença significativa entre o acometimento de homens e mulheres $(6,18,19,22-25)$.

\section{FATORES DE RISCO}

São considerados fatores de risco para MC(28): idade avançada, indivíduos caucasianos, maior exposição aos raios ultravioleta, áreas de MAP com presença de atipia, nevos conjuntivais e síndrome dos nevos atípicos, xeroderma pigmentosum.

O melanoma conjuntival pode originar-se a partir de áreas de MAP com atipia, constituindo $75 \%$ dos casos; de áreas com nevos em cerca de 20\%; e "de novo" (sem lesões prévias) em cerca de 5\% dos $\operatorname{casos}^{(29-31)}$.

A localização mais frequente do MC é a conjuntiva bulbar próxima ao limbo, mas pode ocorrer em qualquer localização, tais como na conjuntiva palpebral, forniceal, na plica ou na região da carúncula ${ }^{(23,25,30,32)}$. MC associados com MAP podem apresentar envolvimento da pele palpebral adjacente ${ }^{(30)}$. O acometimento corneano sem o envolvimento conjuntival ou associado à melanose adquirida da conjuntiva pode ser observado ${ }^{(25,32)}$.

Clinicamente o MC pode ter apresentação variada. Classicamente apresenta-se como uma massa ou uma lesão conjuntival pigmentada elevada. Em alguns casos pode ter aspecto mais difuso ou múltiplo, com bordas mal definidas, particularmente quando associada à MAP(13). Menos comumente, o $\mathrm{MC}$ pode apresentar-se como uma lesão de pigmentação rósea ou avermelhada e até mesmo sem pigmentação (amelanótico), dificultando e retardando seu diagnóstico e tratamento ${ }^{(25)}$

O MC deve ser diferenciado de outras lesões conjuntivais pigmentadas. Os nevos conjuntivais costumam acometer pacientes mais jovens e apresentam-se mobilizáveis juntamente com a conjuntiva sobre a esclera, entretanto, quando presentes em pacientes acima de 40 anos ou na conjuntiva não bulbar, são suspeitos para malignidade. A presença de cistos nos nevos sugere benignidade (Tabela 1). A extensão epibulbar de melanomas uveais ou de melanocitomas devem ser diferenciados de MC baseados em seus achados ultrassonográficos. Uma doença metastática decorrente do melanoma de pele ou de outros tumores (ex., mama ou pulmão), raramente pode acometer a conjuntiva(33).

O MC também deve ser diferenciado de lesões não melanocíticas como: estafiloma, hemorragia subconjuntival, corpo estranho e cistos hemáticos. Pterígios, neoplasia intraepitelial, papilomas ou carcinoma de células escamosas podem apresentar-se com algum grau de pigmentação em indivíduos da raça negra. Melanomas pouco pigmentados ou amelanóticos podem ser confundidos com neoplasias de células escamosas ou com granuloma piogênico. Nos casos de difícil diferenciação, estudos imuno-histoquímicos utilizando marcadores como HMB-45, S-100 ou Ki-67 devem ser realizados pelo patologista ${ }^{(13)}$.

À microscopia óptica quatro tipos celulares de MC podem ser encontrados:

1) Células pequenas poliédricas;

2) Células grandes epitelioides;

3) Células fusiformes;

4) Células em "Balão". Muitos MC são, na verdade, compostos por mais de um tipo celular ${ }^{(10)}$.

\section{ESTADIAMENTO}

De acordo com a sexta classificação TNM da American Joint Committee on Cancer (2002), o MC pode ser estadiado de acordo com sua localização, extensão, o envolvimento de estruturas perioculares (como a invasão de seios nasais e do sistema nervoso central), acometimento de linfonodos regionais (pré-auriculares, parotídeos, submandibulares e cervicais) e metástases à distância. Critérios histopatológicos também são utilizados para determinar a origem tumoral, o envolvimento de linfonodos regionais e metástases à distância ${ }^{(34)}$. Mais recentemente, Damato et al., propuseram um novo sistema de estadiamento clínico para o MC baseado em observações retrospectivas de 40 pacientes. A gradação da severidade da doença pode ser realizada com maior acurácia quando são avaliados os seguintes fatores:

1) a extensão determinada em "minutos de relógio" ou quadrantes;

2) o envolvimento corneano, que não parece correlacionar com severidade;

3) o envolvimento caruncular pode ser associado ao pior prognóstico e aumento da mortalidade;

4) a diferenciação dos níveis de gravidade de acordo com o acometimento "não conjuntival" do MC (por exemplo, a invasão intraocular menos grave do que o envolvimento do SNC);

5) a presença de melanoma in situ (Tis) como precursor de doença invasiva ${ }^{(35)}$. Vide a tabela 2 para maiores detalhes.

\section{TRATAMENTO}

\section{Cirúrgico}

O tratamento de eleição do melanoma maligno conjuntival e da sua lesão precursora, a MAP com atipia, consiste em ressecção cirúrgica de toda a lesão quando possível. A biópsia incisional deve ser evitada devido ao risco de disseminação tumoral e de fibrose local, sendo apenas razoável nos casos associados a MAP com pigmentação difusa e controle em áreas aparentemente (sem pigmentação) e não acometidas ${ }^{(11,36)}$.

A técnica mais comumente empregada é descrita por Shields et al., onde o tumor é excisado com margens de segurança de pelo menos 4-5 mm, evitando sua manipulação direta (técnica "no-touch"(13). A esclerectomia superficial é realizada quando o tumor encontra-se aderido à esclera. Álcool absoluto é aplicado por cerca de 1 minuto no epitélio corneano acometido (margem de segurança de $2 \mathrm{~mm}$ ) e à base da lesão. Crioterapia é aplicada nas margens conjuntivais em 2 ciclos ("double freeze-thaw") e o defeito conjuntival é então corrigido cirurgicamente, utilizando instrumental cirúrgico diferente do utilizado para a exérese do tumor para evitar a disseminação de células tumorais ${ }^{(37)}$.

A utilização da crioterapia como tratamento adjuvante foi primeiramente defendida por Jakobiec na década de $80 \mathrm{com}$ bons resultados ${ }^{(38)}$. A crioterapia oferece tratamento "extra" nas margens 
Tabela 1. Diagnóstico diferencial das lesões melanocíticas conjuntivais

\begin{tabular}{|c|c|c|c|c|c|}
\hline Lesão & Idade ao diagnóstico & Localização & Lateralidade & Características clínicas & Prognóstico \\
\hline Nevos conjuntivais & $\begin{array}{l}\text { Infância } \\
\text { Adolescência } \\
\text { Adultos jovens }\end{array}$ & $\begin{array}{l}\text { Interpalpebral } \\
\text { Região limbar }\end{array}$ & Unilateral & $\begin{array}{c}\text { Indivíduos caucasianos } \\
\text { Margens: bem definidas } \\
\text { Coloração: amarronzado } \\
\text { Mobilidade: sim } \\
\text { Aspecto: plano/elevado com cistos }\end{array}$ & $<1 \%$ evoluem para MC \\
\hline Melanose racial benigna & Adultos & Perilimbar & Bilateral & $\begin{array}{l}\text { Indivíduos raça negra } \\
\text { Margens: mal definidas } \\
\text { Coloração: amarronzado } \\
\text { Mobilidade: sim } \\
\text { Aspecto: plano }\end{array}$ & $\begin{array}{c}\text { Progressão extremamente } \\
\text { rara para MC }\end{array}$ \\
\hline Melanose ocular congênita & $\begin{array}{l}\text { Ao nascimento } \\
\text { Infância }\end{array}$ & Episclera & Unilateral & $\begin{array}{l}\text { Individuos asiáticos } \\
\text { Margens: mal definidas } \\
\text { Coloração: acinzentado } \\
\text { Mobilidade: não } \\
\text { Aspecto: plano }\end{array}$ & $\begin{array}{l}<1 \% \text { evoluem para } \\
\text { melanoma uveal }\end{array}$ \\
\hline Melanose primária adquirida & Adultos de meia idade & $\begin{array}{l}\text { Qualquer localização, } \\
\text { geralmente limbar }\end{array}$ & Unilateral & $\begin{array}{l}\text { Indivíduos caucasianos } \\
\text { Margens: mal definidas } \\
\text { Coloração: amarronzado } \\
\text { Mobilidade: sim } \\
\text { Aspecto: plano }\end{array}$ & $\begin{array}{c}\text { Progressão para MC em } \\
\text { cerca de } 50 \% \text { casos com } \\
\text { MAP com atipia }\end{array}$ \\
\hline Melanoma conjuntival & Idosos & Qualquer localização & Unilateral & $\begin{array}{l}\text { Indivíduos caucasianos } \\
\text { Margens: bem definidas } \\
\text { Coloração: amarronzado } \\
\text { Mobilidade: não } \\
\text { Aspecto: nodular } \\
\text { Vasos nutridores }\end{array}$ & $\begin{array}{l}\text { Metástases em } 26 \% \text { dos } \\
\text { casos em } 10 \text { anos }\end{array}$ \\
\hline
\end{tabular}

da lesão, que muitas vezes pode apresentar a doença microscópica ou sem pigmentação.

A realização da crioterapia é sempre recomendada, porém alguns efeitos indesejáveis podem ocorrer, como fibrose da substância própria conjuntival, perda de cílios, ptose, flacidez palpebral e tarsal, simbléfaro, pseudopterígio, necrose aguda do segmento anterior, atrofia iriana setorial, descompensação corneana, catarata e edema macular cistoide ${ }^{(36)}$.

Damato e Coupland obtiveram resultados satisfatórios utilizando braquiterapia com rutênio e mitomicina $C$ ao invés de crioterapia para pacientes com doença invasiva e MAP, respectivamente ${ }^{(36)}$.

A utilização de membrana amniótica (MA) é util nos casos onde áreas extensas de MAP ou MC precisam ser ressecadas. A MA serve de substrato para a restauração da superfície ocular diminuindo inflamação, cicatrização excessiva e minimizando o risco de deficiência de células tronco limbares ${ }^{(39)}$. A transparência da MA constitui uma das vantagens no monitoramento de recorrências do MC.

A exenteração orbitária está usualmente relacionada a tumores negligenciados e que apresentam fatores de mau prognóstico, como a localização não bulbar (carúncula, por exemplo), maior espessura tumoral e invasão ocular ${ }^{(40)}$. A enucleação é raramente empregada, uma vez que neste procedimento o globo é retirado e a conjuntiva poupada para recobrimento da cavidade.

Toda lesão excisada deve ser obrigatoriamente enviada para análise histopatológica por patologista experiente. O estudo das margens deve ser sempre solicitado. O espécime, antes de colocado na solução de formol, deve ser colocado sobre papel de filtro previamente marcado (exemplo: superior, inferior, nasal e temporal), mantendo a sua orientação anatômica. Isto facilita a identificação correta pelo patologista das margens que possam estar acometidas, norteando futuros tratamentos ${ }^{(37)}$.

\section{Quimioterapia tóPica}

A utilização de quimioterápicos tópicos representa um meio não cirúrgico eficaz, na aplicação de drogas em altas concentrações diretamente na superfície ocular. Sua utilização pode ser indicada em lesões extensas (acometimento límbico maior que 4-5 "horas de relógio") ou multifocais, lesões recorrentes ou na doença microscópica residual (margens positivas identificadas na análise histopatológica ou tumores amelanóticos). É uma alternativa também para pacientes que não concordam com o tratamento cirúrgico. O uso de 5-fluoruracil e de interferon no tratamento das lesões melanocíticas é relatado ${ }^{(41,42)}$. Entretanto, a mitomicina C (MMC) é o quimioterápico de escolha no tratamento primário ou adjuvante de lesões conjuntivais, sendo utilizada preferencialmente na concentração de 0,04\% por um período que pode variar de 1 a 4 semanas. Podem ser necessários de 1 a 3 ciclos para regressão das lesões ${ }^{(43)}$. É importante lembrar que a MMC apresenta grande toxicidade ocular, podendo apresentar efeitos colaterais graves a curto e longo prazo, tais como defeitos epiteliais persistentes, deficiência de células límbicas com conjuntivalização corneana, opacificações ("haze") corneanas focais, catarata, ceratite disciforme, úlceras e "melting" corneano, escleromalácia e perfuração escleral, uveíte anterior e dermatite exfoliante periocular. A oclusão temporária dos pontos lacrimais é recomendada para minimizar sua absorção sistêmica, a irritação da mucosa nasal e a estenose de pontos lacrimais ${ }^{(44)}$. Deficiência de células límbicas a longo prazo é relatada em $23,8 \%$ dos pacientes com MAP com atipia e em $26,7 \%$ dos pacientes com MC tratados com MMC $(45,46)$.

\section{RECORRÊNCIA}

O MC pode recorrer após tratamento em $26 \%$ dos casos em 5 anos, 51\% em 10 anos e 65\% em 15 anos de acompanhamento. A localização do tumor (não acometendo o limbo) e o envolvimento das margens cirúrgicas (margem lateral) são fatores fortemente correlacionados com a recidiva tumoral(25). Outros fatores associados com maior recidiva do MC são invasão palpebral, diâmetro basal maior que $5 \mathrm{~mm}$ e alta densidade linfática intratumoral ${ }^{(47)}$. Tumores recorrentes podem apresentar pigmentação diferente do tumor primário, podendo ser amelanóticos ou de aspecto semelhante a um granuloma piogênico, dificultando o diagnóstico (25).

A eversão das margens palpebrais e o exame minucioso da conjuntiva (mesmo em locais diferentes do sítio primário) devem ser 
Tabela 2. Estadiamento do melanoma maligno conjuntival

Tumor primário $(T)$ - classificação clínica

TX: tumor não pode ser acessado

T0: sem evidência de tumor primário

TI: conjuntiva bulbar

TII: conjuntiva bulbar com envolvimento corneano

TIII: conjuntiva não-bulbar

(tumor estendendo para a conjuntiva forniceal, palpebral ou carúncula)

TIV: extensão para pálpebras, intraocular, órbita, invasão sinusal ou sistema nervoso central
Tis: melanoma in situ

a: bulbar, menos que 1 quadrante

b: bulbar, $>1$ quadrante

c: não-bulbar

d: envolvimento da pele palpebral

TI: conjuntiva bulbar

a: menos que 1 quadrante

b: 1-2 quadrantes

c: $>2$ quadrantes

d: invasão intraocular

TII: envolvimento da conjuntiva palpebral sem acometimento caruncular a: menos que 1 quadrante

b: 1-2 quadrantes

c: $>2$ quadrantes

d: envolvimento da pele palpebral

TIII: conjuntiva palpebral com envolvimento caruncular a: menos que 1 quadrante

b: 1-2 quadrantes

c: $>2$ quadrantes

d: invasão do saco lacrimal

TIV: órbita, invasão sinusal ou intracraniana a: órbita superficial (até equador do globo)

b: órbita profunda (retro-ocular)

c: seios nasais

d: sistema nervoso central

Linfonodos regionais $(\mathrm{N})$

Mantida de acordo com TNM

NX: linfonodos regionais não acessados

N0: ausência de metástases para linfonodos

N1: linfonodos regionais com metástases

Metástases à distância (M)

Mantida de acordo com TNM

MX: metástases não podem ser acessadas

Mo: ausência de metástases à distância

M1: metástases à distância

Tumor primário (pT) - classificação histopatológica

Mantida de acordo com TNM

pTX: tumor primário não acessíve

pT0: ausência de tumor primário

pT1: tumor acometendo apenas epitélio da conjuntiva bulbar

pT2: tumor acometendo conjuntiva bulbar com menos de $0,8 \mathrm{~mm}$ de espessura com acometimento da substância própria

pT3: tumor acometendo conjuntiva bulbar com mais de 0,8 mm de espessura com invasão da substância própria conjuntival ou tumor acometendo pálpebras ou carúncula

pT4: tumor invadindo pálpebras, globo, órbita, seios nasais ou sistema nervoso central

Linfonodos regionais $(\mathrm{pN})$

Mantida de acordo com TNM

pNX: linfonodos regionais não acessados

pN0: ausência de metástases para linfonodos

pN1: linfonodos regionais com metástases

Metástases à distância (pM) Mantida de acordo com TNM

pMX: metástases não podem ser acessadas

pM0: ausência metástases à distância

pM1: metástases à distância

Gradação histológica/origem (G)

Mantida de acordo com TNM

GX: origem não pode ser acessada

G0: melanose adquirida primária sem atipia celular

G1: nevo conjuntival

G2: melanose adquirida primária com atipia celular (acometimento epitelial somente)

G3: "de novo" 
rotineiramente realizados e qualquer pigmentação"nova"em paciente submetido à exérese tumoral prévia deve ser tratada como recorrência com quimioterapia tópica, crioterapia ou uma nova cirurgia.

\section{METÁSTASES}

As taxas de metástase encontradas por Shields et al., para o MC foram de 16\% em 5 anos, 26\% em 10 anos e 32\% em 15 anos de acompanhamento. Os locais mais comumente afetados pela doença metastática são os linfonodos regionais, seguidos do cérebro, fígado, pulmões e da doença disseminada. Os fatores relacionados a maiores taxas de metástase foram o envolvimento da margem lateral na peça cirúrgica e a localização não acometendo o limbo ${ }^{(25)}$. A palpação dos linfonodos regionais deve ser realizada de rotina nas consultas de seguimento oftalmológico. Uma equipe multidisciplinar, com envolvimento de um oncologista é recomendada para a detecção precoce da doença metastática.

\section{LINFONODO SENTINELA}

A investigação e a biópsia dos chamados "linfonodos sentinelas" (ou regionais) é controversa, porém vem sendo recomendada por alguns autores. Os linfonodos regionais podem ser o sítio clínico inicial de metástases em $45 \%$ dos casos. Tumores menores de $2 \mathrm{~mm}$ de espessura possuem aproximadamente $5 \%$ de incidência acumulativa em 10 anos para metástases, tanto regionais quanto sistêmicas. Em contrapartida, tumores mais espessos que $2 \mathrm{~mm}$ ou não limbares apresentam incidência cumulativa em 5 anos de aproximadamente 20\% para doença metastática regional ou sistêmica ${ }^{(48)}$.

\section{MORTALIDADE}

A mortalidade em pacientes acometidos por MC é aproximadamente de $30 \%$ em 10 anos. Folberg et al., reportaram uma taxa de mortalidade geral de $26 \%$. Os seguintes fatores podem ser associados com maior taxa de mortalidade em pacientes com MC originados em áreas de MAP:

1) Presença de invasão pagetoide na região de MAP do tumor;

2) Envolvimento da conjuntiva palpebral;

3) Presença de atipia moderada ou severa na região de MAP do tumor; e

4) Ausência ou escassez de células poliédricas na porção de MAP do tumor. Nenhum destes fatores foi associado a um pior prognóstico nos MC originados em áreas não-MAP(30).

Tumores de localização não bulbar, de tipo celular misto, com invasão linfática, ou multifocais, apresentando aumento de espessura (>4 mm) e de diâmetro (>5 mm) tumoral representam fatores de mau prognóstico associados a maiores taxas de mortalidade ${ }^{(47,49)}$. Em centros terciários de referência, a mortalidade associada ao MC pode ser um pouco mais baixa ocorrendo com taxas de $7 \%$ em 5 anos e de 13\% em 10 anos. Fatores de mau prognóstico incluem sintomas iniciais (presença de nódulos) e achados histopatológicos de melanoma "de novo" sem associação com MAP(25).

\section{CONCLUSÕES}

Apesar de raro, o melanoma maligno conjuntival é extremamente agressivo com elevadas taxas de metástases e de mortalidade. A identificação de lesões pigmentadas benignas e precursoras é fundamental e o seguimento destes pacientes com exames periódicos deve ser realizado. Um exame oftalmológico minucioso e a documentação fotográfica contribuem para uma detecção precoce de mudanças clínicas sugestivas de transformação maligna. A excisão cirúrgica seguindo critérios de segurança é imprescindível para o tratamento adequado evitando a doença residual, e diminuindo assim as taxas de recorrência e de metástase. A avaliação histopatológica acessando fatores prognósticos é crucial. A avaliação por oftalmologista experiente e uma equipe multidisciplinar são peças chave no sucesso do manejo destes pacientes.

\section{REFERÊNCIAS}

1. Grossniklaus HE, Green WE, Luckenbach M, Chan CC. Conjunctival lesions in adults. A clinical and histopathologic review. Cornea. 1987:6(2):78-116. Review.

2. Paridaens AD, MacCartney AC, Hungerford JL. Multifocal amelanotic conjunctival melanoma and acquired melanosis sine pigmento. Br J Ophthalmol. 1992;76(3):163-5.

3. Jay B. Naevi and melanomata of the conjunctiva. Br J Ophthalmol. 1965:49:169-204.

4. Folberg R. Jakobiec FA, Bernardino VB, Iwamoto T. Benign conjunctival melanocytic lesions. Clinicopathologic features. Ophthalmology. 1989;96(4):436-61.

5. Shields CL, Fasiuddin AF, Mashayekhi A, Shields JA. Conjunctival nevi: clinical features and natural course in 410 consecutive patients. Arch Ophthalmol. 2004;122(2): 167-75. Erratum: Arch Ophthalmol. 2006;124(2):198. Fasiudden, Airey [corrected to Fasiuddin, Airaj F].

6. Novais GA, Fernandes BF, Belfort RN, Castiglione E, Cheema DP, Burnier MN Jr. Incidence of melanocytic lesions of the conjunctiva in a review of 10,675 ophthalmic specimens. Int J Surg Pathol. 2010;18(1):60-3

7. Cameron ME. Spontaneous cyclical pigmentary changes in a conjunctival naevus. $\mathrm{Br}$ J Ophthalmol. 1982;66(2):115-7.

8. Liesegang TJ, Campbell RJ. Mayo Clinic experience with conjunctival melanomas. Arch Ophthalmol.1980;98(8):1385-9.

9. Gerner N, Norregaard JC, Jense OA, Prause JU. Conjunctival naevi in Denmark 19601980. A 21-year follow-up study. Acta Ophthalmol Scand. 1996;74(4):334-7.

10. Jakobiec FA, Folberg R, Iwamoto T. Clinicopathologic characteristics of premalignant and malignant melanocytic lesions of the conjunctiva. Ophthalmology. 1989; 96(2):147-66

11. Shields JA, Shields CL, Mashayekhi A, Marr BP, Benavides R, Thangappan A, et al. Primary acquired melanosis of the conjunctiva: risks for progression to melanoma in 311 eyes. The 2006 Lorenz E. Zimmerman lecture. Ophthalmology. 2008;115(3):511-9 e2.

12. Baum TD, Adamis AP, Jakobiec FA. Primary acquired melanosis of the conjunctiva. Int Ophthalmol Clin. 1997;37(4):61-72.

13. Shields J. Eyelid, conjunctival, and orbital tumors: atlas and text book. $2^{\text {th }}$ ed. Philadelphia: Lippincott Williams \& Wilkins; 2008

14. Folberg R, McLean IW, Zimmerman LE. Primary acquired melanosis of the conjunctiva. Hum Pathol. 1985;16(2):129-35.

15. Heindl LM, Hofmann-Rummelt C, Adler W, Bosch JJ, Holbach LM, Naumann GO, et al. Tumor-associated lymphangiogenesis in the development of conjunctival melanoma. Invest Ophthalmol Vis Sci. 2011:52(10):7074-83.

16. Scotto J, Fraumeni JF Jr., Lee JA. Melanomas of the eye and other noncutaneous sites: epidemiologic aspects. J Natl Cancer Inst. 1976;56(3):489-91.

17. Char DH. The management of lid and conjunctival malignancies. Surv Ophthalmol. 1980;24(6):679-89.

18. Seregard S, Kock E. Conjunctival malignant melanoma in Sweden 1969-91. Acta Ophthalmol (Copenh). 1992:70(3):289-96.

19. Lommatzsch PK, Lommatzsch RE, Kirsch I, Fuhrmann P. Therapeutic outcome of patients suffering from malignant melanomas of the conjunctiva. $\mathrm{Br} \mathrm{J}$ Ophthalmol. 1990;74(10):615-9.

20. Inskip PD, Devesa SS, Fraumeni JF Jr. Trends in the incidence of ocular melanoma in the United States, 1974-1998. Cancer Causes Control. 2003.14(3):251-7. Comment in: Cancer Causes Control. 2004;15(1):95-6; author reply 101-2.

21. Yu GP, Hu DN, McCormick S, Finger PT. Conjunctival melanoma: is it increasing in the United States? Am J Ophthalmol. 2003;135(6):800-6.

22. Norregaard JC, Gerner N, Jensen OA, Prause JU. Malignant melanoma of the conjunctiva: occurrence and survival following surgery and radiotherapy in a Danish population. Graefes Arch Clin Exp Ophthalmol. 1996;234(9):569-72.

23. Missotten GS, Keijser S, De Keizer RJ, De Wolff-Rouendaal D. Conjunctival melanoma in the Netherlands: a nationwide study. Invest Ophthalmol Vis Sci, 2005; 46(1):75-82.

24. Anastassiou G, Heiligenhaus A, Bechrakis N, Bader E, Bornfeld N, Steuhl KP. Prognostic value of clinical and histopathological parameters in conjunctival melanomas: a retrospective study. Br J Ophthalmol. 2002;86(2):163-7. Comment in: Br J Ophthalmol. 2002;86(2):127.

25. Shields CL, Shields JA, Gündüz K, Cater J, Mercado GV, Gross N, et al.. Conjunctiva melanoma: risk factors for recurrence, exenteration, metastasis, and death in 150 consecutive patients. Arch Ophthalmol. 2000;118(11):1497-507. Comment in: Arch Ophthalmol. 2000;118(11):1525-33

26. McDonnell JM, Carpenter JD, Jacobs P, Wan WL, Gilmore JE. Conjunctival melanocytic lesions in children. Ophthalmology. 1989;96(7):986-93.

27. Strempel I, Kroll P. Conjunctival malignant melanoma in children. Ophthalmologica. 1999;213(2):129-32.

28. Seregard S. Conjunctival melanoma. Surv Ophthalmol. 1998;42(4):321-50.

29. Folberg R, McLean IW. Zimmerman LE. Conjunctival melanosis and melanoma. Ophthalmology. 1984;91(6):673-8 
30. Folberg R, McLean IW, Zimmerman LE. Malignant melanoma of the conjunctiva. Hum Pathol. 1985;16(2):136-43.

31. Spencer WH. Ophthalmic pathology and the American Academy of Ophthalmology. Ophthalmology. 1996;103(8 Suppl):S109-17.

32. Jeffrey IJ, Lucas DR, McEwan C, Lee WR. Malignant melanoma of the conjunctiva. Histopathology. 1986;10(4):363-78.

33. Tuomaala S, Aine E, Saari KM, Kivelä T.Corneally displaced malignant conjunctiva melanomas. Ophthalmology. 2002;109(5):914-9.

34. Sobin LH, Witterkind C. TNM Classification of malignant tumours. New York: Wiley-Liss; 2002

35. Damato B, Coupland SE. An audit of conjunctival melanoma treatment in Liverpool. Eye (Lond). 2009;23(4):801-9.

36. Shields CL, Shields JA. Tumors of the conjunctiva and cornea. Surv Ophthalmol. 2004; 49(1):3-24.

37. Shields JA, Shields CL, De Potter P. Surgical management of conjunctival tumors. The 1994 Lynn B. McMahan Lecture. Arch Ophthalmol. 1997;115(6): 808-15.

38. Jakobiec FA, Rini FJ, Fraunfelder FT, Brownstein S. Cryotherapy for conjunctival primary ac quired melanosis and malignant melanoma. Experience with 62 cases. Ophthalmology. 1988;95(8):1058-70

39. Espana EM, Prabhasawat P, Grueterich M, Solomon A, Tseng SC. Amniotic membrane transplantation for reconstruction after excision of large ocular surface neoplasias. B J Ophthalmol. 2002;86(6):640-5. Comment in: Br J Opthalmol. 2002;86(12):1460.

40. Paridaens AD, McCartney AC, Minassian DC, Hungerford JL. Orbital exenteration in 95 cases of primary conjunctival malignant melanoma. Br J Ophthalmol. 1994;78(7):520-8.

41. de Keizer RJ, de Wolff-Rouendaal D, van Delft JL. Topical application of 5-fluorou- racil in premalignant lesions of cornea, conjunctiva and eyelid. Doc Ophthalmol. 1986;64(1):31-42.

42. Herold TR, HintschichC. Interferon alpha for the treatment of melanocytic conjunctival lesions. Graefes Arch Clin Exp Ophthalmol. 2010;248(1):111-5.

43. Chalasani R, Giblin M, Conway RM. Role of topical chemotherapy for primary acquired melanosis and malignant melanoma of the conjunctiva and cornea: review of the evidence and recommendations for treatment. Clin Experiment Ophthalmol. 2006; 34(7):708-14.

44. Abraham LM, Selva D, Casson R, Leibovitch I. Mitomycin: clinical applications in ophthalmic practice. Drugs. 2006:66(3):321-40.

45. Lichtinger A, Pe'er J, Frucht-Pery J, Solomon A. Limbal stem cell deficiency after topical mitomycin $C$ therapy for primary acquired melanosis with atypia. Ophthalmology. 2010:117(3):431-7.

46. Ditta LC, Shildkrot Y, Wilson MW. Outcomes in 15 patients with conjunctival melanoma treated with adjuvant topical mitomycin C: complications and recurrences. Ophthalmology. 2011;118(9):1754-9.

47. Heindl LM, Hofmann-Rummelt C, Adler W, Bosch JJ, Holbach LM, Naumann GO, et al. Prognostic significance of tumor-associated lymphangiogenesis in malignant melanomas of the conjunctiva. Ophthalmology. 2011;118(12):2351-60.

48. Tuomaala S, Kivela T. Metastatic pattern and survival in disseminated conjunctival melanoma: implications for sentinel lymph node biopsy. Ophthalmology. 2004; 111(4):816-21.

49. Paridaens $A D$, Minassian DC, McCartney $A C$, Hungerford JL.Prognostic factors in primary malignant melanoma of the conjunctiva: a clinicopathological study of 256 cases. Br J Ophthalmol. 1994;78(4):252-9.

\title{
VI Congresso Brasileiro da \\ VI Congresso Brasileiro da \\ Sociedade Brasileira de Lentes de Contato, Córnea e Refratometria - SOBLEC
}

\author{
2 a 4 de maio de 2013
}

\section{Centro de Convenções Frei Caneca}

São Paulo (SP)

Informações:

Tels.: (11) 5084-9174/5082

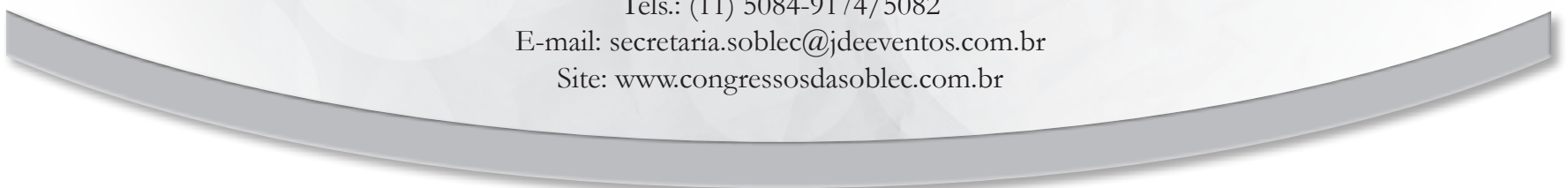

